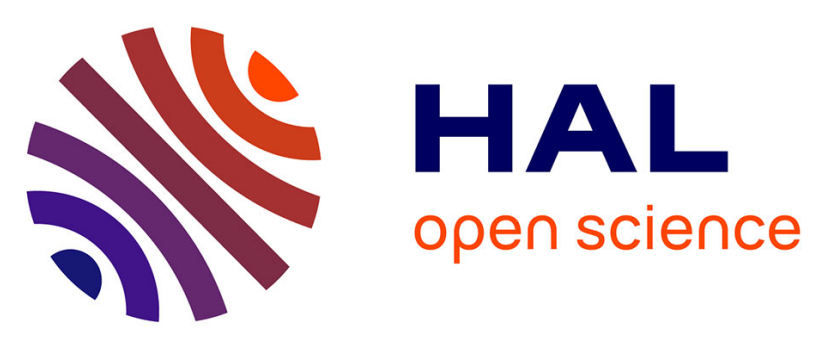

\title{
Electron delocalization in mixed-valence Keggin polyoxometalates. Ab initio calculation of the local effective transfer integrals and its consequences on the spin coupling.
}

Nicolas Suaud, Alejandro Gaita-Arino, Juan Modesto Clemente-Juan, Jose Marin-Sanchez, Eugenio Coronado

\section{To cite this version:}

Nicolas Suaud, Alejandro Gaita-Arino, Juan Modesto Clemente-Juan, Jose Marin-Sanchez, Eugenio Coronado. Electron delocalization in mixed-valence Keggin polyoxometalates. Ab initio calculation of the local effective transfer integrals and its consequences on the spin coupling.. Journal of the American Chemical Society, 2002, 124, pp.15134. hal-00003331

\section{HAL Id: hal-00003331 https://hal.science/hal-00003331}

Submitted on 19 Nov 2004

HAL is a multi-disciplinary open access archive for the deposit and dissemination of scientific research documents, whether they are published or not. The documents may come from teaching and research institutions in France or abroad, or from public or private research centers.
L'archive ouverte pluridisciplinaire HAL, est destinée au dépôt et à la diffusion de documents scientifiques de niveau recherche, publiés ou non, émanant des établissements d'enseignement et de recherche français ou étrangers, des laboratoires publics ou privés. 


\section{Electron delocalization in mixed-valence Keggin}

polyoxometalates. Ab initio calculation of the local effective transfer integrals and its consequences on the spin coupling.

Nicolas Suaud*, Alejandro Gaita-Ariño, Juan Modesto Clemente-Juan, José Sánchez-Marín and Eugenio Coronado*. Instituto de Ciencia Molecular, Universidad de Valencia, C/ Doctor Moliner 50, 46100 Burjassot, Spain. email: Nicolas.Suaud@uv.es, Eugenio.Coronado@uv.es 


\section{Abstract}

We present a quantitative evaluation of the influence of the electron transfer on the magnetic properties of mixed-valence polyoxometalates reduced by two electrons. For that purpose, we extract from valence-spectroscopy $a b$ initio calculations on embedded fragments, the value of the transfer integrals between $W$ nearest-neighbor atoms in a mixed-valence $\alpha P W_{12} O_{40}$ polyoxowolframate Keggin anion. In contradiction with what is usually assumed, we show that the electron transfer between edge-sharing and corner-sharing $W O_{6}$ octahedra have very close values. Considering fragments of various ranges, we analyse the accuracy of calculations on fragments based on only two $W O_{5}$ pyramids which should allow a low cost general study of transfer parameters in polyoxometalates. Finally, these parameters are introduced in an extended Hubbard hamiltonian that models the whole anion. It permits to prove that electron transfers induce a large energy gap between the singlet ground state and the lowest triplet states providing a clear explanation of the diamagnetic properties of the mixed-valence Keggin ions reduced by two electrons. 


\section{INTRODUCTION}

Polyoxometalates (POM) are a class of inorganic compounds with a remarkable degree of molecular and electronic tunabilities that impact in disciplines as diverse as catalysis [1], medicine [2] and materials science [3]. These compounds are molecular metal oxides mainly based on $V, M o$ and $W$ ions in their highest oxidation states. Due to the cluster structure, POM are specially useful as model systems for the studies of magnetic and electronic interactions. Indeed, many of these structures allow the inclusion of well-isolated clusters of paramagnetic ions with various nuclearities and definite topologies and geometries [4]. On the other hand, they permit controlled injection of electrons, giving rise to mixed-valence species in which delocalized electrons may coexist and interact with localized magnetic moments. In this context they provide unique systems for the development of new theories in the mixed valence area.

Some typical structural types are the Lindquist [5] $\left(M_{6} O_{19}^{2-}, M=M o, W\right)$, Keggin [6] $\left(X M_{12} O_{40}^{m-}, X=P, S i, C o, N i, \ldots\right)$ and Dawson-Wells [7] $\left(P_{2} M_{18} O_{62}^{n-}\right)$. The Keggin structure is constituted of four edge-sharing triads of $M O_{6}$ octahedra arranged around the $X$ atom in such a way that the resulting species has a tetrahedral symmetry. The Keggin structure can also be viewed as formed by a $\mathrm{XO}_{4}^{m-}$ clathrate encapsulated into a neutral $\mathrm{M}_{12} \mathrm{O}_{36}$ sphere based on corner sharing $M O_{5}$ square-pyramids whose apical oxygen atoms points outside the sphere [8,9] (see Fig. 1a). They are thus sometime noted as $\mathrm{XO}_{4}^{m-} \subset M_{12} O_{36}$.

An important property of the polyoxometalates anions is that their identity is usually preserved by reversible redox processes [10], forming "heteropoly blues" or "heteropoly browns" reduction products by addition of various electrons which are delocalized over the sphere [11]. Experimentally it has been found that when the reduced species contain an even number of delocalized electrons, their spins are always completely paired, even at room temperature. This result is general and has been found not only in the Keggin structure but also in the other ones. It was initially attributed to a very strong antiferromagnetic coupling via a multiroute superexchange mechanism [12], but more recently it has been theoretically shown that a combination of electron repulsion and electron delocalization can also stabilize the singlet ground state [13-15].

The extended-Hubbard model Hamiltonian used for these calculations handles with the effective parameters corresponding to the main microscopic interactions: 
- the $t$ electron transfer (hopping) parameter between corner-sharing $M O_{6}$ octahedra ,

- the $t^{\prime}$ electron transfer (hopping) parameter between edge-sharing octahedra ,

- the $U$ on-site electron repulsion between electrons belonging to the magnetic orbital of a same metal center,

- the five $V_{1} \ldots V_{5}$ inter-site electron repulsion corresponding to the five inequivalent pairs of metal centers.

Using this model it may be possible to qualitatively explain the strong antiferromagnetic coupling between the pair of electrons in the reduced Keggin anion. However, the space spanned by this number of parameters is by far too large to permit an univoque solution of the problem. In order to reduce the size of this space and thus to draw a picture of the coupling between the two delocalized electrons, independent information on the values of these parameters is essential. The main aim of the present article is to evaluate theoretically some of these parameters. To reach this goal we will calculate the transfer parameters $t$ and $t^{\prime}$ by using very accurate $a b$ initio methods. These values will be then introduced in the extended Hubbard model in order to obtain information on the lowest lying spin levels of the system and on the effective coupling between the pair of electrons that are delocalized over the Keggin structure.

\section{EMBEDDED FRAGMENTS}

The transfer (hopping) effective integrals are essentially local parameters [16]. Therefore fragment spectroscopy calculations can be used to accurately evaluate their values. The fragments are embedded in a bath adapted to reproduce the main effects of the rest of the crystal, namely, the short-range Pauli exclusion and the long-range Madelung potential. This bath consists in a large number of punctual charges and total-ion pseudopotentials [17] (TIP's). A quasi-spherical embedding of punctual charges is obtained by replacing all the atoms surrounding the fragment (those closer than $20 \AA$ from the center of the considered Keggin anion) by punctual charges. The total-ion pseudopotentials TIP's are put in the position of all the atoms of the first and second shells enclosing the fragment. In references $[18,19]$ a complete description of embedded fragment spectroscopy calculations is reported as well as a discussion of the accuracy of the embedding procedure. 
The biggest fragment of the Keggin cluster on which we have performed calculations is represented by the $\mathrm{PW}_{4} \mathrm{O}_{20}$ tetramer (Figure 1b). This fragment is based on four adjacent $W O_{6}$ octahedra and on the $\mathrm{PO}_{4}$ encapsulated clathrate. $W 1$ and $W 2$ belong to the same triad (and thus belong to edge-sharing $W O_{6}$ octahedra); $W 3$ and $W 4$ are part of the same triad (and thus belong to edge-sharing $W O_{6}$ octahedra) adjacent to the triad of $W 1$ and $W 2$. $W 1$ and $W 4$, on one side, and $W 2$ and $W 3$, on the other side, belong to corner-sharing octahedra. Such a fragment thus permits the electron delocalization along corner-sharing octahedra ( $t$ parameter), along edge-sharing octahedra ( $t^{\prime}$ parameter $)$ and between second neighbors octahedra ( $t^{d}$ parameter).

Figure 1c represents the $W_{4} O_{16}$ fragment, extracted from the precedent $P W_{4} O_{20}$ fragment by substituting the $\mathrm{PO}_{4}$ molecule by the appropriated punctual charges and TIP's. It is thus composed of four corner-sharing $W_{5}$ pyramids. A comparison between the values of the transfer parameters obtained on the $\mathrm{PW}_{4} \mathrm{O}_{20}$ and $W_{4} O_{16}$ fragments permits to evaluate the role that the $\mathrm{PO}_{4}$ plays on the electron delocalization. This is so because punctual charges and TIP's do not support any basis set, and thus can not held any bridging pathway between $W$ centers. Therefore, assuming that punctual charges and TIP's mimic properly the electrostatic and Pauli exclusion effects, the role of the $\mathrm{PO}_{4}$ on the electron delocalization is the difference between the values of the transfer parameters calculated on the $P W_{4} O_{20}$ and on the $W_{4} O_{16}$ fragments.

The smallest fragments we have considered are formed by the dimeric units $W_{2} O_{9}$ and $W_{2} O_{10}$ represented in figure $1 \mathrm{~d}$. In these three cases the $W, O$ and $P$ ions that belong to the $4 \mathrm{~W}$-based fragments but not to the $2 \mathrm{~W}$-based fragments are modelled by punctual charges and TIP's added to the total embedding. The two $W_{2} O_{9}$ fragments, based on corner-sharing pyramids, are extracted from edge-sharing octahedra (rightmost fragment) and corner-sharing octahedra (fragment on the left). The two pyramids of the $W_{2} O_{10}$ fragment do not share any atom. These fragments afford the independent evaluation of $t^{\prime}, t$ and $t^{d}$ parameters, respectively. Thus, a comparison with the previous results should permit to check the accuracy of these calculations.

As the structure of the $P W_{12} O_{40}$ cluster only changes slightly when the charge compensating cations of the salt are changed, all the calculations reported in this work are based on the X-ray crystallographical coordinates [8] of the $\left(\mathrm{H}_{5} \mathrm{O}_{2}^{+}\right)_{3}\left(\mathrm{PW}_{12} \mathrm{O}_{40}^{3-}\right)$ salt. In this case, the $T_{d}$ symmetry of the polyoxometalate makes all the $\mathrm{W}$ atoms equivalent. On the one hand 
this high symmetry permits much quicker calculations. On the other hand, the equivalence of all the $W$ ions induce the equivalence of the interactions $W 1 \leftrightarrow W 2$ and $W 3 \leftrightarrow W 4$ (along edge-sharing octahedra) as well as the interactions $W 1 \leftrightarrow W 4$ and $W 2 \leftrightarrow W 3$ (along corner-sharing octahedra) and of the interactions $W 1 \leftrightarrow W 3$ and $W 2 \leftrightarrow W 4$ between second neighbor octahedra.

\section{MODEL HAMILTONIANS AND EXTRACTION OF PARAMETERS}

In the reduced Keggin ion, the unpaired electrons are essentially delocalized over the $d_{x y}$-like orbital of each of the $W$ ions (pointing in between the equatorial $O$ ions of the octahedron). Hence, a model hamiltonian that takes into account the transfer (hopping) parameter between $d_{x y}$-like orbitals of adjacent $W$ ions and the Coulomb repulsion between the two extra electrons is well suited to reproduce the electron delocalization and spin coupling in this system [14]. We focus in this article on the calculation of the transfer parameters. The extraction of the effective electrostatic repulsions from similar $a b$ initio calculations will be the aim of a forthcoming paper. For an evaluation of these parameters based on pure electrostatic consideration, see reference [14].

\section{A. 4- $W$-ions based fragments}

Let us consider one electron delocalized over the 4 - $W$-ions based fragments (fragments $1 \mathrm{~b}$ and 1c). The model hamiltonian induces the following doublet states:

$$
\begin{aligned}
& \Psi_{1}=\frac{d_{1}+d_{2}+d_{3}+d_{4}}{2} \\
& \Psi_{2}=\frac{d_{1}-d_{2}-d_{3}+d_{4}}{2} \\
& \Psi_{3}=\frac{d_{1}+d_{2}-d_{3}-d_{4}}{2} \\
& \Psi_{4}=\frac{d_{1}-d_{2}+d_{3}-d_{4}}{2}
\end{aligned}
$$

where $d_{1}, d_{2}, d_{3}$ and $d_{4}$ stand for the Slater determinants constructed when the extra electron is situated on $W 1, W 2, W 3$ and $W 4$, respectively.

The transfer parameters are thus written as: 


$$
\begin{gathered}
t=\left\langle d_{1}|H| d_{4}\right\rangle=\left\langle d_{2}|H| d_{3}\right\rangle \\
t^{\prime}=\left\langle d_{1}|H| d_{2}\right\rangle=\left\langle d_{3}|H| d_{4}\right\rangle \\
t^{d}=\left\langle d_{1}|H| d_{3}\right\rangle=\left\langle d_{2}|H| d_{4}\right\rangle
\end{gathered}
$$

The transition energies are:

$$
\begin{aligned}
& E_{1 \rightarrow 2}=E_{2}-E_{1}=-2 t^{\prime}-2 t^{d} \\
& E_{1 \rightarrow 3}=E_{3}-E_{1}=-2 t-2 t^{d} \\
& E_{1 \rightarrow 4}=E_{4}-E_{1}=-2 t-2 t^{\prime}
\end{aligned}
$$

where $E_{1}, E_{2}, E_{3}$ and $E_{4}$ are respectively the energy of the states $\Psi_{1}, \Psi_{2}, \Psi_{3}$ and $\Psi_{4}$; $E_{1 \rightarrow 2}, E_{1 \rightarrow 3}$ and $E_{1 \rightarrow 4}$ being respectively the transition energy between the states $\Psi_{1}$ and $\Psi_{2}, \Psi_{1}$ and $\Psi_{3}, \Psi_{1}$ and $\Psi_{4}$.

The transfer parameters are extracted from the comparison of the transition energies calculated by ab-initio methods with equations 8,9 and 10 .

\section{B. $2 \mathrm{~W}$-ions based fragments}

Each one of the three $2 \mathrm{~W}$-ions based fragments (Fig. 1d) supports only one kind of electron transfer. Thus, the fragment on the left is used for extracting $t$, while the rightmost one allows the evaluation of $t^{\prime}$; finally, from the fragment at the bottom we can extract the value of $t^{d}$.

The transition energy $E_{D D}$ between symmetric and antisymmetric doublet states predicted by the model hamiltonian is thus related to the transfer parameter by the relation:

$$
E_{D D}=E_{D_{+}}-E_{D_{-}}=2 t^{i}
$$


where $t^{i}$ stands for $t, t^{\prime}$ or $t^{d}$ depending on the fragment we are dealing with. $E_{D_{+}}$and $E_{D_{-}}$are the energy of the doublet states $\Psi_{D_{+}}$and $\Psi_{D_{-}}$, respectively. The symmetry of these two functions are those of $d_{1}+d_{2}$ and $d_{1}-d_{2}$. Thus, for the $W_{2} O_{9}$ fragments $\Psi_{D_{+}}$is the antisymmetric doublet and $\Psi_{D_{-}}$is the symmetric one, whereas in the $W_{2} O_{10}$ fragment $\Psi_{D_{+}}$is the symmetric doublet and $\Psi_{D_{-}}$is the antisymmetric one.

Hence, the energies of the symmetric and antisymmetric doublet states obtained by $a b$ initio calculations directly give the values of the transfer parameters.

\section{COMPUTATIONAL METHODOLOGY}

On each of the previously described fragments we performed both CASSCF and CASPT2 calculations using the MOLCAS suite of programs [20].

According to the physics of mixed-valence compounds that permits to differentiate the orbitals depending of their contribution to electron transfer, the CASSCF procedure divides the molecular orbitals into three subspaces:

- the inactive orbitals that remain doubly occupied;

- the active orbitals whose occupation is allowed to change;

- the virtual orbitals that remain unoccupied.

The Complete Active Space (CAS) is then defined, for a given number of active electrons (those occupying the active orbitals), as the set of all the Slater determinants that can be built according to the previous occupation rules. The CASSCF consists in the self-consistent optimization of all the orbitals and of the coefficients of the wave function developped on the CAS. Polarization and correlation of the electrons of the active orbitals are thus variationally taken into account in the mean field of the electrons of the inactive orbitals.

From this zeroth-order CASPT2 goes further and takes into account the dynamical polarization and correlation effects in a second-order perturbative treatment, that is as a sumation of interactions that do not interact between them. Even if CASPT2 is more precise than CASSCF, we present both results. Indeed, the differences between the two methods account for the influence of the dynamical effects on the transfer parameters providing at the same time a checking of the suitability of the CASSCF to be a good zeroth-order for CASPT2. 
Difference Dedicated Configuration Interaction [21, 22] (DDCI) is a more accurate method but often requires much larger calculations than CASPT2. In the DDCI method the dynamical polarization and correlation effects are taken into account variationally still only those effects that contribute (at the second-order of perturbation) to the energy differences between the states of the CAS are taken into account. This method requires the diagonalization of the matrix representative of the DDCI space generated by all the following excitations on all the determinants of the CAS:

- 1-hole (one electron is excited from an inactive orbital to an active orbital),

- 1-particule (active $\rightarrow$ virtual),

- 1-hole-1-particule (inact. $\rightarrow$ virt.),

- 2-holes (inact.+inact. $\rightarrow$ active+active),

- 2-particules (act.+act. $\rightarrow$ virt.+virt.),

- 2-holes-1-particule (inact.+inact. $\rightarrow$ act.+virt.),

- 1-hole-2-particules (inact.+act. $\rightarrow$ virt.+virt.).

Besides the excitations within the DDCI space, other double excitations are also possible, the 2-holes-2-particules excitations which by the way are the most numerous (for a schematic representation of the determinants of the DDCI space and of the 2-holes-2-particules excitations of the CAS, see Figure 2). If we only consider energy differences, these out of space excitations can be ignored when a common set of Molecular Orbitals (MO) are used for all the calculations [21]. Indeed in this case their effect is only to shift the energy of all the states of the CAS by the same energy (at the second-order of perturbation). All the calculations were thus performed on the set of MO optimized at the CASSCF level for the lowest doublet state.

Due to the size of the matrices to diagonalize, the DDCI calculations have only been performed on the $2 \mathrm{~W}$-based fragments in order to obtain results as accurate as possible and to compare these results with those obtained with the CASPT2 method. The DDCI results were obtained with the CASDI suite of programs [23]. 
In our system, the active orbitals are the $d_{x y}$-like orbital of the $W$ ions. The CAS is thus based on one electron and 4 orbitals in the case of $4 \mathrm{~W}$-based fragment calculations (the corresponding states are those of section III A); it is reduced to one electron and 2 orbitals in the case of $2 \mathrm{~W}$-based fragment calculations.

In all the calculations, the inner-core electrons ([1 $\left.s^{2} 2 s^{2} 2 p^{6} 3 s^{2} 3 p^{6} 4 s^{2} 3 d^{10} 4 p^{6} 5 s^{2} 4 d^{10} 4 f^{14}\right]$ for the $W$ atoms and $\left[1 s^{2}\right]$ for the $O$ and $P$ atoms) are represented by effective core potentials (ECP). The outer-core and valence electrons are represented using a $13 s 10 p 9 d 5 f$ primitive basis set contracted to $3 s 3 p 4 d 2 f$ for the $W$, a $5 s 6 p 1 d$ primitive basis set contracted to $2 s 4 p 1 d$ for the $O$ atoms that belong to the coordination sphere of the $W$ treated in the fragments, a $5 s 6 p 1 d$ primitive basis set contracted to $1 s 2 p 1 d$ for the other $O$ atoms (the two $O$ the $P O_{4}$ molecule not linked to the $W$ of the fragments) and a $7 s 6 p 1 d$ primitive basis set contracted to $1 s 1 p 1 d$ for the $P$ atom. Exact expressions of the basis sets and ECP can be found in reference [24].

\section{RESULTS AND DISCUSSION}

\section{A. Results on 4- $W$-ions fragments}

The results obtained on the $4-W$-ions fragments (fragments b and c in figure 1 ) are summarized in table I.

As it is generally observed in mixed-valence compounds $[16,18,19]$, we can observe that the dynamical effects (the difference between CASPT2 and CASSCF results) remain small and do not change significantly the values of the transfer parameters.

In contrast with what may be expected, $t$ and $t^{\prime}$ have very similar values. This is a surprising result as the $W-O-W$ angle between the two $W$ ions and the bridging oxygen (the oxygen that does not belong to the $\mathrm{PO}_{4}$ molecule) has very different values. For edgesharing octahedra this angle is $127^{\circ}$ and for corner-sharing octahedra it is $153^{\circ}$, whereas the $W-O$ distance have very close values, $1.964 \AA$ and $1.957 \AA$ respectively. Thus, this angle seems not to be the only relevant parameter involved in the electron transfer. It would be interesting to get a better understanding of all the relevant geometrical parameters that act on the electron transfer integrals and can explain why $t \simeq t^{\prime}$. Calculations in this context are presented in the subsection $\mathrm{VC}$. 
The value of $t^{d}$ is about 4 times smaller with respect to those of $t$ and $t^{\prime}$, and of the same sign.

Finally, the very small differences between the results extracted on the $P W_{4} O_{20}$ and $W_{4} O_{16}$ fragments, less than $15 \mathrm{meV}$, permit to extract two important conclusions:

- the bridging effects due to the $O$ anions of the $\mathrm{PO}_{4}$ molecule are very small, as it could have been expected due to the large distance $(\simeq 2.5 \AA)$ between these anions and the $W$ atoms. Note that the electron transfer pathways from one $W$ atom to the other through the $\mathrm{PO}_{4}$ molecule are allowed in the $\mathrm{PW}_{4} \mathrm{O}_{20}$ fragment but not in the $W_{4} O_{16}$ fragment.

- the effects of the atoms of the $\mathrm{PO}_{4}$ molecule on the transfer integrals are accurately reproduced by the choosen charges and TIP's. Whereas in the $W_{4} O_{16}$ fragment these atoms are modelled by punctual charges and TIP's, they are treated with basis set in the $\mathrm{PW}_{4} \mathrm{O}_{20}$ fragment.

These results clearly show the aptness of calculations on four corner-sharing pyramids embedded fragment but, even for this reduced fragment, the calculations are too large to treat systems where the symmetry between $W$ ions is reduced. Another problem arises from the meaning of the $t^{d}$ parameter. Indeed, as we extracted three parameters from three energy differences, we could not check the aptness of this parameter to model the system.

We show in the next section how calculations on dimer fragments permit to lift these two problems.

\section{B. Dimer calculations}

The results of the calculations performed on the three $2 \mathrm{~W}$-based fragments are presented in table II.

We can see that the results on $t$ and $t^{\prime}$ compare very well with the results extracted from the $W_{4} O_{16}$ fragment with differences smaller than $3 \%$ at the CASSCF level as well as at the CASPT2 level. On the one hand, this confirms that, as usual in mixed-valence compounds, the electron transfer in the Keggin anion is essentially a local parameter and can be accurately calculated on fragments based on the interacting metal ions and on the 
atoms of their coordination sphere. On the other hand, these results proof that the effects of the $\mathrm{WO}_{5}$ pyramids on the atoms of the fragment are correctly reproduced by the choosen punctual charges and TIP's. As the same punctual charges and TIP's were used in the whole embedding, we thus can assume that the whole embedding correctly reproduce the main effects of the crystal on the fragments [19] (checkings of the aptness of an embedding based on a limited number of punctual charges to reproduce the Madelung field will be presented in a forthcoming paper [25] in the case of another polyoxometalate). Hence, the dimer fragment can be considered a quite good model to extract the transfer parameters in a very efficient and simple way.

Concerning $t^{d}$, we can see that the values extracted from dimer calculations are significantly different from those extracted from $4 \mathrm{~W}$-based fragments, $-102 \mathrm{meV}$ versus $-125 \mathrm{meV}$ at the CASPT2 level. Indeed, whereas in the two other fragments all the closest neighbors of the bridging $O$ anions are in the fragment, the environment of the bridging $O$ anions in this case is not so accurately treated since part of the closest neighbors of these anions are modelled by charges and TIP's. Nevertheless, the dimer calculations prove that the $t^{d}$ parameter has a non-negligible value and justificate our choice to take this parameter into account in the model hamiltonian. The $t^{d}$ value obtained from $4 \mathrm{~W}$-based fragment is not an artifact due to the extraction of three parameters from three transition energies and we can trust its value.

Thus, accurate values of electron tranfers can be obtained from calculations on correctly embedded small fragments based only on two $\mathrm{WO}_{5}$ pyramids.

\section{Influence of the $W-O-W$ angle on the transfer parameter}

We have shown that those $O$ ions shared by the octaedra and the $\mathrm{PO}_{4}$ molecule do not support any pathway for the electron transfer. Thus, the delocalization only occurs through the orbitals of the other $O$ ions shared by two octaedra. We can wonder if the $W-O-W$ angle formed by the later oxygen and the tungsten ions is the only relevant parameter for the electron transfer, if it can explain why the values of $t$ and $t^{\prime}$ only differ by about $40-50 \mathrm{meV}$, and if it could be possible to predict the value of these parameters (or at least the ratio between them) only from these crystallografic data.

We have demonstrated that the $2 \mathrm{~W}$-based fragments are well designed for extracting the 
values of $t$ and $t^{\prime}$. Thus we will use these fragments to get more informations on the effect of the $W-O-W$ angle on the values of $t$ and $t^{\prime}$. For that purpose, we performed calculations on model fragments obtained by rotating each of the $W_{5}$ pyramids around the bridging $O$ atoms in the plane containing these atoms and the two $W$ atoms. 20 model fragments were formed (ten for the tranfer between edge-sharing octaedra and ten for the tranfer between corner-sharing octaedra) corresponding to variations of the $W-O-W$ angles of $-5^{\circ},-4^{\circ}$, $-3^{\circ},-2^{\circ},-1^{\circ},+1^{\circ},+2^{\circ},+3^{\circ},+4^{\circ}$ and $+5^{\circ}$ around the real angles given by the $\mathrm{x}$-ray structure $\left(152.4^{\circ}\right.$ for corner-sharing $W O_{6}$ octaedra and $126.8^{\circ}$ for edge-sharing octaedra). We did not perform calculation on more distorted fragments as they should be too far from the real structure to give relevant values. The results are represented in figure 3.

We observe that when the $W-O-W$ angle increases the transfer parameters increase in absolute value. This is due to the increase of the overlap between the magnetic orbital of the $W$ ions and the bridging orbital of the $O$ ion. Such a variation is of the same order of magnitude for corner and edge-sharing fragments with a tangent at $152.4^{\circ}$ of about $-4.6 \mathrm{meV} /{ }^{\circ}$ and of about $-3.9 \mathrm{meV} /{ }^{\circ}$ at $126.8^{\circ}$ (at the CASPT2 level).

If the $W-O-W$ angle was the only geometrical parameter acting on the transfer parameter, model fragments based either on corner-sharing octaedra or edge-sharing octaedra should give the same value for the transfer parameter when the $W-O-W$ angle is the same. Extrapolations of the curves presented in figure 3 clearly show that this is not the case.

Thus, other structural parameters than the $W-O-W$ angle have strong effects on the electron transfer and should be taken into account either to explain why the values of $t$ and $t^{\prime}$ are close or to roughly predict the values of the electron transfer parameters in other polyoxometalate clusters.

\section{Comparison between CASPT2 and DDCI results.}

We have now established the validity of $2 \mathrm{~W}$-based fragments comparing, at the same level of calculations, the values for the transfer parameters extracted from fragments of various nuclearities. It is thus possible on these small fragments to go further than the perturbative treatment of the dynamical effects (CASPT2 method) and perform a variational calculation of the transfer parameters with the DDCI methods. Since the 
2W-based fragments calculations give a good evaluation of the order of magnitude of the $t^{d}$ parameter but with errors of about $20 \%$, it is useless to intend to perform more accurate calculations on such a fragment (and it is at the moment technically impossible to obtain DDCI results on $4 \mathrm{~W}$-based fragments). We thus only performed DDCI calculations to evaluate the $t$ and $t^{\prime}$ parameters which are the only relevant ones in the theoretical models proposed to describe the magnetic behavior of two-electron-reduced Keggin ions [14]. The results are the following:

$$
t=-467 m e V \quad t^{\prime}=-507 m e V
$$

These are the most accurate results presented in this work. Differences with the CASPT2 calculations (on the same fragments) are very small, of the order of $20 \mathrm{meV}$, less than $5 \%$.

Thus, the transfer electron between $W$ of corner-sharing $W_{6}$ octahedra and between $W$ of edge-sharing octahedra have similar intensity, whatever the level of calculation and the fragment used for these calculations.

\section{E. Prediction of the Singlet-Triplet energy gap.}

Theoretical models [14] were previously developped to predict, in function of the transfer and electrostatic repulsion parameters, the magnetic behaviour of a two-electron-reduced Keggin anion. Figure 4 represents the energy levels of the supported singlet and triplet lowest states.

The values of the transfer integrals obtained from $a b$ initio calculations give a ratio $t^{\prime} /|t| \simeq$ -1.086 (vertical dashed line in Figure 4). Using these results the model predicts a singlet ${ }^{1} A_{1}$ ground state and that the first excited state is a ${ }^{3} T_{2}$ triplet state. The energy gap between these states is thus about $0.6 t$, that is $280 \mathrm{meV}$. This very large value permits to understand the diamagnetic properties of two-electron-reduced Keggin anion compounds [12].

\section{CONCLUSION}

In this article we have quantitatively shown for the first time how the electron hopping occurring in mixed-valence Keggin polyoxometalates can promote a strong antiferromagnetic coupling between the pair of delocalized electrons. 
To reach this goal we have combined the exact results obtained from a phenomenological extended Hubbard type model that allows to evaluate the effect of the electron transfer and electron repulsion on the properties of the whole mixed-valence cluster, with ab initio calculations that provide accurate values of the transfer parameters from calculations on small fragments of the Keggin cluster. These fragments permit large configuration interaction DDCI calculations, and thus comparison with multireference second-order pertubative theory CASPT2 results, providing at the same time a general and efficient method to extract these parameters in all the polyoxometalate structures.

As a perspective, we want to focus on the computational resources needed for this work. Whereas the calculations on $4 \mathrm{~W}$-based fragment were only possible thanks to the power of the center of calculation of the University of Valencia, those performed on dimer fragments (at CASSCF, CASPT2 and DDCI levels) requires less then $10 \mathrm{~Gb}$ of hard disk, 300Mb of memory and about 30 hours on a 866Mhz-PIII "home" computer. Our work thus intents to be the grounding of a low cost general study of the transfer parameters in mixed-valence polyoxometalates compounds, and in other kinds of high nuclearity mixed-valence system.

\section{Acknowledgments}

This research was financially supported by European Community (Network Molnanomag, $\mathrm{n}^{\circ}$ HPRN-CT-1999-00012), by the spanish Misterio de Ciencia y Technologia (MAT20013507) and by the Generalitat Valenciana (GV01-312). We thank Marie-Bernadette Lepetit for many fruitful discussions.

[1] I. V. Kozhevnikov, Chem. Rev. 98, 171 (1998); N. Mizuno and M. Misono, Chem. Rev. 98, 199 (1998); M. Sadakane and E. Steckhan, Chem. Rev. 98, 219 (1998).

[2] J. T. Rhule, C. L. Hill, D. A. Judd and R. F. Schinazi, Chem. Rev. 98, 327 (1998).

[3] A. Müller, F. Peters, M. T. Pope and D. Gatteschi, Chem. Rev. 98, 239 (1998); E. Coronado and C. J. Gómez-García, Chem. Rev. 98, 273 (1998); W. G. Klemperer and C. G. Wall, Chem. Rev. 98, 297 (1998); M. T. Pope and A. Müller, "Polyoxometalates: From Platonic Solids to Anti-Retroviral Activity", Kluwer Academic Publishers: Dordrecht, The Netherlands, 1994. 
[4] J. M. Clemente-Juan and E. Coronado, Coord. Chem. Rev. 193-195, 193 (1999); J. M. Clemente-Juan and E. Coronado, Coord. Chem. Rev. XX, 361 (1999);

[5] I. Lindquist, Arkiv. Kemi. 5, 247 (1952).

[6] J. F. Keggin, Nature 131, 908 (1933).

[7] B. Dawson, Acta Cryst. 6, 113 (1953).

[8] G. M. Brown, M.-R. Noe-Spirlet, W. R. Busing and H. A. Levy, Acta Cryst. B 33, 1038 (1977).

[9] C. Rocchiccioli-Deltcheff, R. Thouvenot and R. Franck, Spectrochim. Acta 32A, 587 (1976); R. Acerete, N. Casañ-Pastor, J. Bas-Serra and L. C. W. Baker, J. Am. Chem. Soc. 111, 6049 (1989); L. P. Kazansky and B. R. McGarvey, Coord. Chem. Rev. 188, 157 (1999); J. M. Maestre, X. Lopez, C. Bo, J.-M. Poblet and N. Casañ-Pastor, J. Am. Chem. Soc. 123, 3749 (2001); X. López, J. M. Maestre, C. Bo and J.-M Poblet, J. Am. Chem. Soc. 123, 9571 (2001).

[10] N. Casañ-Pastor, P. Gomez-Romero, G. B. Jameson and L. C. W. Baker, J. Am. Chem. Soc. 113, 5658 (1991).

[11] M. T. Pope, Isopoly and Heteropoly Metalates, Springer-Verlag, Berlin, 1983.

[12] M. Kozik, C. F. Hammer, and L. C. W. Baker, J. Am. Chem. Soc. 108, 2748 (1986); M. Kozik, and L. C. W. Baker, J. Am. Chem. Soc. 109, 3159 (1987); M. Kozik, N. Casañ-Pastor, C. F. Hammer, and L. C. W. Baker, J. Am. Chem. Soc. 110, 1697 (1988); M. Kozik, and L. C. W. Baker, J. Am. Chem. Soc. 112, 7604 (1990). N. Casañ-Pastor, and L. C. W. Baker, J. Am. Chem. Soc. 114, 10384 (1992);

[13] S. A. Borshch and B. Bigot, Chem. Phys. Letters 212, 398 (1993).

[14] J. J. Borrás-Almenar, J. M. Clemente-Juan, E. Coronado and B. S. Tsukerblat, Chem. Phys. 195, 1 (1995).

[15] J. J. Borrás-Almenar, J. M. Clemente-Juan, E. Coronado and B. S. Tsukerblat, Chem. Phys. 195, 17 (1995); J. J. Borrás-Almenar, J. M. Clemente-Juan, E. Coronado and B. S. Tsukerblat, Chem. Phys. 195, 29 (1995).

[16] I. de P. R. Moreira, F.Illas, C. J. Calzado, J. F. Sanz, J. P. Malrieu, N. Ben Amor and D. Maynau, Phys. Rev. B 59, 6593 (1999).

[17] P. Durand et al, Theor. Chim. Acta 38, 283 (1975).

[18] C. Jimenez Calzado, J. Fernandez Sanz, J. P. Malrieu and F. Illas, Chem. Phys. Lett. 307, 
102 (1999); D. Munoz, F. Illas, I. de P.R. Moreira, Phys. Rev. Letters 84, 1579 (2000); C. Jimenez Calzado, J. Fernandez Sanz and J. P. Malrieu, J. Chem. Phys. 112, 5158 (2000).

[19] N. Suaud and M.-B. Lepetit, Phys. Rev. B 62, 402 (2000); N. Suaud and M.-B. Lepetit, Phys. Rev. Lett. 88, 056404 (2002).

[20] MOLCAS suite of programs. K. Anderson, M. P. Fülscher, G. Karlström, R. Lindh, P. A. Malqvist, J. Olsen, B. Roos, A. J. Sadlej, M. R. A. Blomberg, P. E. M. Siegbahn, V. Kello, J. Noga, M. Urban, P. O. Widmark, MOLCAS Version 4, Dpt of Theor. Chem., Chem. Center, Univ. of LUND, P. O. B. 124, S-221 00 Lund, Sweden, Lund 1994.

[21] J. P. Malrieu, J. Chem. Phys. 47, 4555 (1967).

[22] R. Broer and W.J.A. Maaskant, Chem. Phys. 102, 103 (1986); J. Miralles, O. Castell, R. Caballol and J. P. Malrieu, Chem. Phys. 172, 33 (1993); J. Cabrero, N. Ben Amor, C. de Graaf, F. Illas and R. Caballol, J. Phys. Chem. A 104, 9983 (2000).

[23] CASDI suite of programs. D. Maynau, N. Ben Amor and J. V. Pitarch-Ruiz, University of Toulouse, France (1999); N. Ben Amor and D. Maynau, Chem. Phys. Letters 286, 211 (1998); J. V. Pitarch-Ruiz, J. Sánchez-Marín and D. Maynau, J. Comp. Chem, in press.

[24] Z. Barandiarán and L. Seijo, Can. J. Chem. 70, 409 (1992).

[25] C. Jimenez Calzado, A. Gaita-Ariño, N. Suaud and Eugenio Coronado, to be published. 


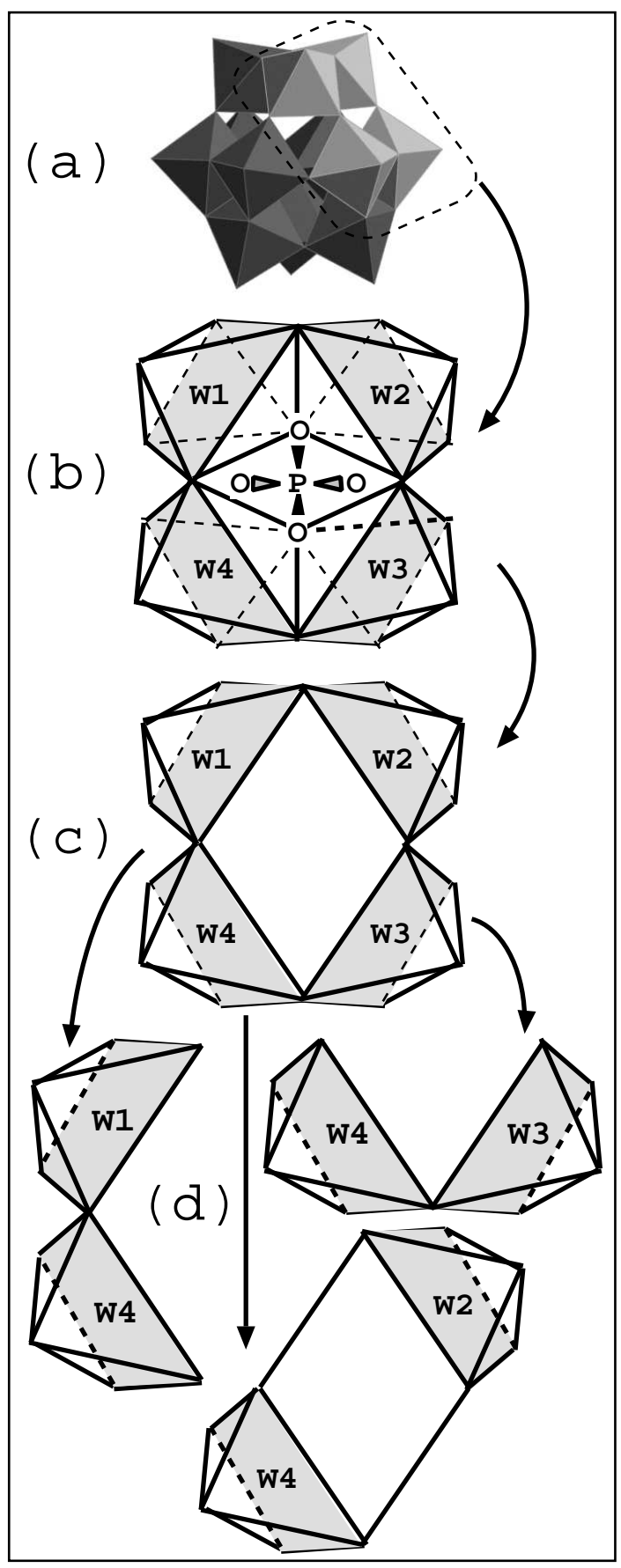

Figure 1: (a) Schematic structure of a Keggin anion. (b) The $P W_{4} O_{20}$ fragment. (c) The $W_{4} O_{16}$ fragment. (d) The three $2 \mathrm{~W}$-based fragments. The oxygen atoms occupy the corners of the octahedra or pyramids. The atoms modelled by punctual charges and TIP's are not represented. 

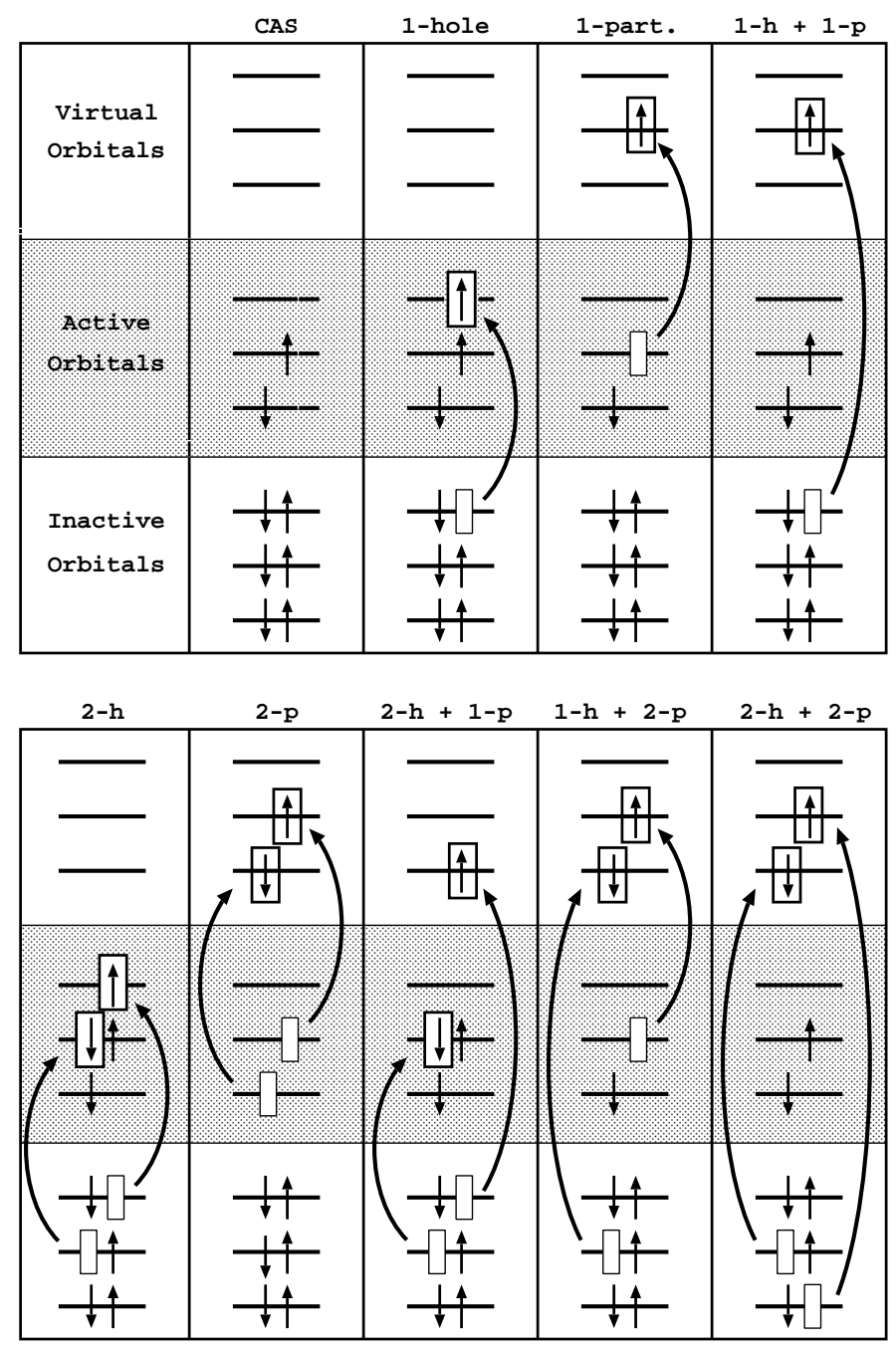

Figure 2: Schematic representation of the single and double excitations on the CAS. The space spanned by $\{$ CAS, 1-h, 1-p, 1-h+1-p, 2-h, 2-p, 2-h+1-p, 1-h+2-p $\}$ correspond to the DDCI space. The number of orbitals and electrons in the figure is arbitrary. 


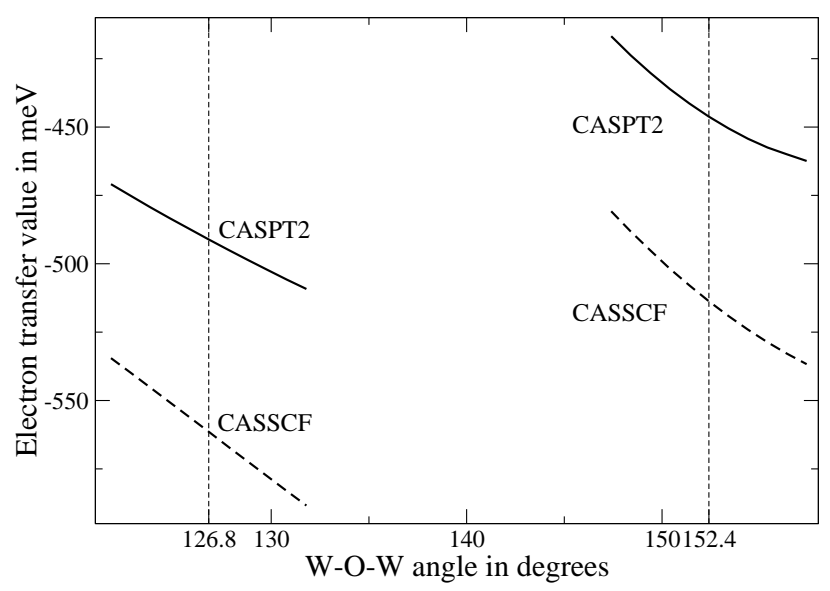

Figure 3: Variations of the value of the electron transfer parameter with the $W-O-W$ angle around the real structure. The curves on the left represent the variation obtained from edge-sharing fragments, the curves on the right represent the variation obtained from corner-sharing fragments.

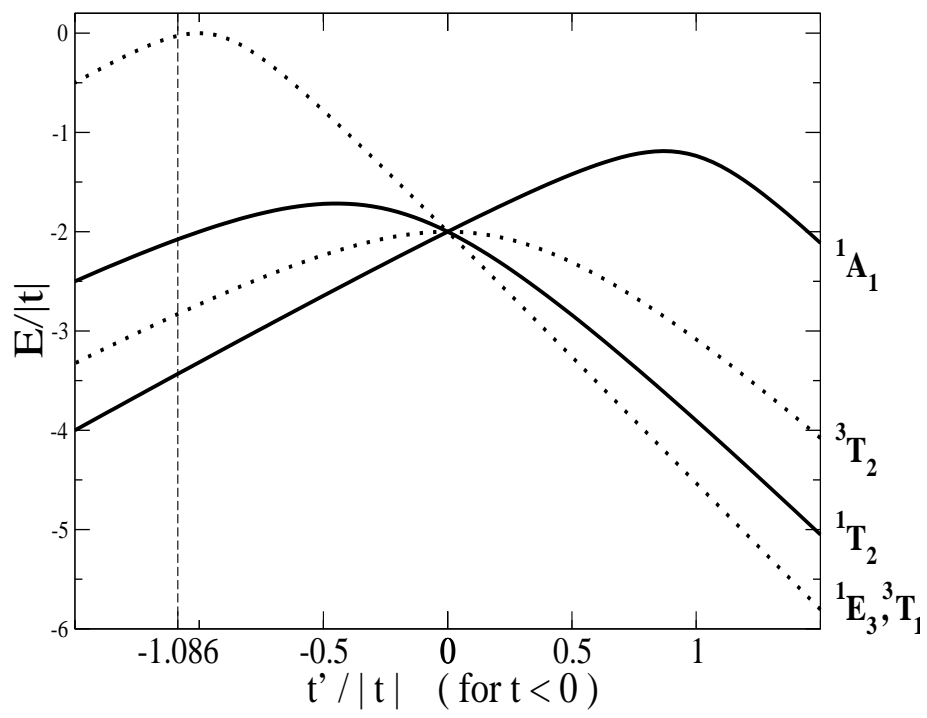

Figure 4: Theoretical energy levels of the doubly reduced Keggin ion. The dotted lines stands for triplet states, the solid lines for singlet states (see ref. [14] for the denomination of the states). The vertical dashed line represents the value of the calculated $t^{\prime} /|t|$ ratio. 


\begin{tabular}{|c|c|c|c|}
\hline & & $P W_{4} O_{20}$ & $W_{4} O_{16}$ \\
\hline \multirow{2}{*}{ t } & CASSCF & -506 & -510 \\
\hline & CASPT2 & -428 & -443 \\
\hline \multirow{2}{*}{$t^{\prime}$} & CASSCF & -551 & -551 \\
\hline & CASPT2 & -470 & -479 \\
\hline \multirow{2}{*}{$t^{d}$} & CASSCF & -87 & -89 \\
\hline & CASPT2 & -123 & -125 \\
\hline
\end{tabular}

Table I: Results in meV of the calculations on the $4 \mathrm{~W}$-based fragments.

\begin{tabular}{ccc}
\hline \hline & & \\
& CASSCF & CASPT2 \\
\hline$t^{2}$ & -510 & -445 \\
$t^{\prime}$ & -560 & -490 \\
$t^{d}$ & -80 & -102 \\
\hline
\end{tabular}

Table II: Results in meV of the calculations on the $2 \mathrm{~W}$-based fragments. 